\title{
INFRARED OPTICAL AND X-RAY DETERMINATION OF PARAMETERS FOR MOVPE GROWN InAs $\mathrm{s}_{1-x} \mathrm{Sb}_{x}$ EPILAYERS
}

\author{
B. MarszaleK*, E. Zielińska-Rohozińska, R. Bożek, R. StęPniewski \\ AND A.M. WITOWSKI
}

Institute of Experimental Physics, Warsaw University, Hoża 69, 00-681 Warsaw, Poland

The experimental room-temperature transmission of metalorganic vapour phase epitaxy grown InAsSb epilayers is compared with calculations based on a Kane model of the band structure. The band structure parameters are found. The composition of the samples was determined by X-ray diffraction.

PACS numbers: 71.20.-b, 78.20.-e, 78.30.-j

The ternary semiconductor $\operatorname{InAs} s_{1-x} \mathrm{Sb}_{x}$ is of great interest because of its potential application for long wavelength infrared photodetectors [1]. Its energy gap as a function of the composition shows strong bowing and goes well below the energy gap of InSb. This makes it possible to obtain detectors that can operate at $300 \mathrm{~K}$ for wavelengths longer than $10 \mu \mathrm{m}$. The advantage of $\mathrm{In} \mathrm{As}_{1-x} \mathrm{Sb}_{x}$ over $\mathrm{Hg}_{1-x} \mathrm{Cd}_{x} \mathrm{Te}$ lies in its high stability, very good semiconductor properties, and possible use of industry standard technologies (MBE and MOVPE). For layers grown by these techniques a strong ordering was observed [2-4], which can lead to the band-gap reduction as observed in other III-V compounds $[4,5]$. From a comparison between the energy gap data obtained on MBE samples from photoluminescence and photoconductivity experiments and the data obtained on bulk crystals from transmission measurement the ordering-induced reduction of the band-gap of $45 \mathrm{meV}$ was found [3]. It is expected [4] that for MOVPE grown samples the ordering is stronger than for an MBE material. Therefore, using samples grown by this method, we performed careful studies of room temperature (RT) IR transmission to find changes of the energy gap due to the expected ordering. Usually the photon energy for which the absorption coefficient has a given value (e.g. $\left.300 \mathrm{~cm}^{-1}[6,7]\right)$ is taken as the value of energy gap. This method is not justified, because the shape of the interband absorption spectrum is different for different energy gaps and different Fermi energies. In the present work the band structure parameters were established from the comparison of the measured infrared transmission with the calculated one.

*Also Teachers College of Physics in Faculty of Physics, Warsaw University. 
All InAs $s_{1-x} \mathrm{Sb}_{x}$ epitaxial layers investigated in this paper were grown in a horizontal, atmospheric pressure pressure MOVPE reactor using trimethylindium, trimethylantimony and $0.5 \%$ solution of arsine in hydrogen. The substrates were semi-insulating GaAs (001) without any intentional disorientation. The composition of the layers was determined by X-ray diffraction, from comparison of positions of the diffraction peaks from InAsSb and from GaAs substrate, assuming a linear dependence of the lattice constant versus composition. The X-ray measurements also allow a check of the crystal quality (rocking curve between 240 and 600 arc$\mathrm{sec}$ ). The thickness of the layer was measured using an electron microscope and was found to be between 3 and $5 \mu \mathrm{m}$.

In the investigated As concentration range one expects to observe an interesting nonlinear dependence of energy gap on composition [6,7]. We compare the measured transmission with that obtained theoretically the Kane model [8] in the large spin-orbit splitting approximation and thus established the band structure parameters. To calculate transmission, we start from the dynamic dielectric function (DDF) $\varepsilon(\omega)=\varepsilon_{\text {inf }}+\varepsilon_{86}(\omega)$. The real part of dielectric function $\varepsilon_{86}(\omega)$ due to interband transitions from $\Gamma_{8}$ heavy hole (with effective mass $0.5 m_{\mathrm{e}}$ [9]) to $\Gamma_{6}$ conduction band was calculated numerically (see e.g. [10]). The optical parameters (index of refraction and extinction coefficient) were obtained from the DDF and used to calculate the transmission of a thin InAsડ̃b layer on GaAs substrate. The transmission was calculated using a model described elsewhere [11], taking into account multiple reflections, but not interference phenomena. We assume a lossless substrate with a refraction index independent of photon energy and equal to 3.4. In such a case the reflection coefficient for the In AsSb/GaAs interface in less than $5 \%$ and the interference fringes due to the interference of light in the epilayer are very weak. The results of the calculations were compared with experimental IR transmission data. Varying the values of the energy gap $E_{\mathrm{g}}$ and matrix element $P$, the theoretical curve was found, which best describes the experimental points. The Fermi energy used in transmission calculations was taken from transport measurements data. Sometimes small adjustment of this value was necessary, suggesting that the conductivity is not fully related to "bulk" carriers in the layer. As an example, in Fig. 1 the experimental IR transmission of InSb sample and sample with $36 \%$ of As are compared with theoretical calculations in the energy range where interband transitions occur. As one can see the agreement is very good. One can also see structures due to two-phonon absorption and reststrahlen in the substrate. In Fig. 2 we present the energy gap as function of composition. The expected bowing of $E_{\mathrm{g}}$ vs. $x[6,7]$ is clearly seen. It can be described using a quadratic equation (solid curve in Fig. 2):

$$
E_{\mathrm{g}}(\mathrm{eV})=0.658 x^{2}-0.834 x+0.35 \text {. }
$$

For comparison, the previously obtained dependence [7] is also plotted (dashed line) in Fig. 2, with values obtained in [4]. As one see, our values are smaller than those reported earlier and the discrepancy is greater for smaller $E_{\mathrm{g}}$. Our findings agree very well with the data from [4] for an MBE material. Therefore we suggest that the differences are due to the method of determining the energy gap and not due to the ordering effect. It should be pointed out that the steepness of absorption edge is less for smaller energy gaps and is more affected by the positions of the 




Fig. 1. Transmission of $\mathrm{InAs}_{0.36} \mathrm{Sb}_{0.64}$ (circles) and $\mathrm{InSb}$ (squares) measured at room temperature. The structure on the low energy side is due to the GaAs substrate reststrahlen and two-phonon absorption. Solid lines represent transmission calculated for interband transitions from the Kane model.

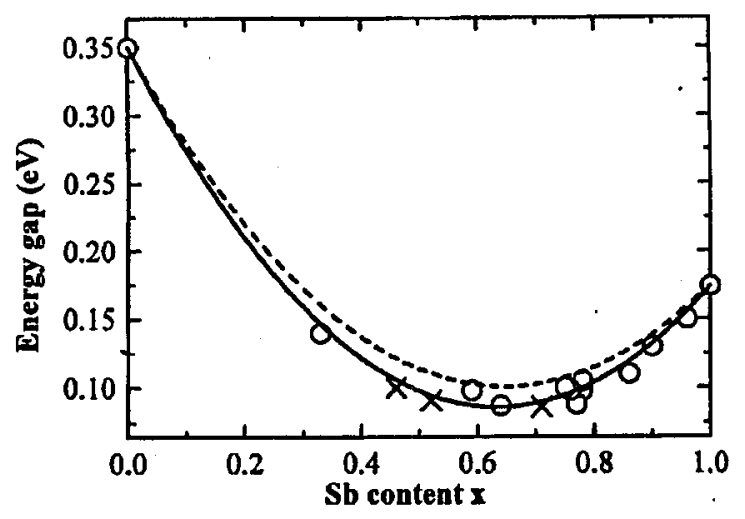

Fig. 2. The dependence on antimony content $x$ of the energy gap $\left(E_{\mathrm{g}}\right)$. Circles represent data obtained in this work, the solid line is given by the quadratic equation fitted to our points, the dashed line is taken after [7] and crosses represent data from [4] - see text for details.

Fermi energy. The value of the absorption used for determining the gap $\left(300 \mathrm{~cm}^{-1}\right)$ is therefore found at energies removed further from $E_{\mathrm{g}}$ than for large gap crystals.

The method presented in our work is more justified than the usual procedure of determining $E_{\mathrm{g}}$ as the photon energy for an arbitrarily chosen value of the absorption coefficient. No change of the energy gap due to the probable or- 
dering was observed. Our data shows that the ternary compound can be used for photodetectors that can operate up to wavelengths of $15 \mu \mathrm{m}$.

\section{Acknowledgments}

The authors want to express their gratitude to J. Eusakowski for valuable discussions, to M. Szydłowski for his help in measuring X-ray diffraction and M. Sadowski for careful reading of the manuscript. We would also like to thank R.A. Stradling for making his data available to us prior to publication. This research was partially supported by the Committee for Scientific Research grant 7 T08A 00209.

\section{References}

[1] A. Rogalski, Prog. Quantum Electron. 13, 191 (1989).

[2] H.R. Jen, K.Y. Ma, G.B. Stringfellow, Appl. Phys. Lett. 54, 1154 (1989).

[3] S.R. Kurtz, L.R. Dawson, R.M. Biefeld, D.M. Follstaed, B.L. Doyle, Phys. Rev. B 46, 1909 (1992).

[4] R.A. Stradling, Acta Phys. Pol. A 92, 851 (1997).

[5] Su-Huai Wei, Wlex Zunger, Appl. Phys. Lett. 58, 2684 (1991).

[6] C.J. Wolley, J. Warner, Can. J. Phys. 42, 1879 (1964).

[7] M.Y. Yen, B.F. Levine, C.G. Bethea, K.K. Choi, A.Y. Cho, Appl. Phys. Lett. 50, 927 (1987).

[8] E.O. Kane, J. Phys. Chem. Solids 1, 249 (1957).

[9] C.R. Pidgeon, R.N. Brown, Phys. Rev. 146, 575 (1966).

[10] W. Szuszkiewicz, A.M. Witkowski, M. Grynberg, Phys. Status Solidi B 87, 637 (1979).

[11] C.A. Hougen, J. Appl. Phys. 66, 3763 (1989). 